\title{
Anti-NMDA Receptor Paraneoplastic Encephalitis: An Important Differential Diagnosis in Subacute Psychosis
}

\author{
André Amaral Gomes ${ }^{\mathrm{a}}$, Elika Pinho ${ }^{\mathrm{a}}$, Ana Vaz ${ }^{\mathrm{b}}$, Pedro Castro ${ }^{\mathrm{c}}$, João Santos Antunes ${ }^{\mathrm{a}}$, \\ Eduarda Pereira $^{\mathrm{b}}$, Celeste Dias ${ }^{\mathrm{b}}$, Fernando Friões $^{\mathrm{a}}$, Jorge Almeida ${ }^{\mathrm{a}}$
}

\begin{abstract}
Anti NMDA (N-methyl D-aspartate) receptor encephalitis has been recently described in young women with ovarian teratoma. Memory and psychiatric disturbances are frequent followed by respiratory and autonomic failure warranting long intensive care admissions. We report on a female patient with paraneoplastic encephalitis manifested by behavioural changes and neurological impairment, followed by respiratory and hemodynamic instability. Anti-NMDA receptor antibodies were found in blood and spinal fluid in association with an imamature grade 2 ovarian teratoma. She made a full recovery after complete oophorectomy and institution of intravenous immunoglobulin and corticosteroids. Anti-NMDA encephalitis should be considered in any female patient presenting with de novo psychiatric symptoms. As in other paraneoplastic syndrome, its therapy is based in resection of the underlying tumor associated with immunomodulators. Timely initiation of therapy may significantly improve outcome as in this case.
\end{abstract}

Keywords: Encephalitis; NMDA; Paraneoplastic; Receptor; Teratoma; Psychosis

\section{Introduction}

A syndrome comprised of memory deficits, psychiatric symptoms, decreased level of consciousness and hypoventi-

Manuscript accepted for publication November 15, 2012

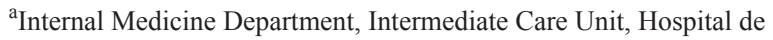
São João, Porto, Portugal

${ }^{\mathrm{b}}$ Intensive Medicine Department, Neurocritical Care Unit, Hospital de São João, Porto, Portugal

${ }^{\mathrm{c}}$ Neurology Department, Hospital de São João, Porto, Portugal

${ }^{\mathrm{d}}$ Corresponding author: Andre Amaral Gomes, Intermediate Care

Unit - Internal Medicine Department - Hospital de São João, Porto, Al.

Prof. Hernani Monteiro, 4200-319 Porto, Portugal.

Email: andreamaralgomes@gmail.com

doi: http://dx.doi.org/10.4021/jmc986w lation was reported in four young women with ovarian teratomas in 2005 [1, 2]. Soon after, specific autoantibodies to the $\mathrm{N}$-methyl D-aspartate receptor (NMDAR) were detected in a cohort of twelve patients with the same symptoms [2]. The discovery of this disorder, termed anti NMDAR encephalitis, has become a topic of interest in the medical community $[3,4]$, changing the diagnostic approach to clinical problems as diverse as subacute memory disturbance, abnormal movements or seizures and limbic encephalitis of unknown aetiology.

Despite the severity of the clinical manifestations, patients usually respond well to timely immunotherapy (corticosteroids, intravenous immunoglobulin or plasma exchange) and tumour resection, with more than $75 \%$ of patients showing substantial recovery $[5,6]$. We report the case of one patient with anti-NMDAR encephalitis.

\section{Case Report}

A 32-year-old Caucasian woman with past medical history of ovarian cysts was brought to the psychiatric emergency department with complaints of behavioural change namely anxiety and insomnia, followed by short-term memory loss and oro-facial dyskinesias with 2 weeks duration. Three days before admission she began experiencing visual and audi-

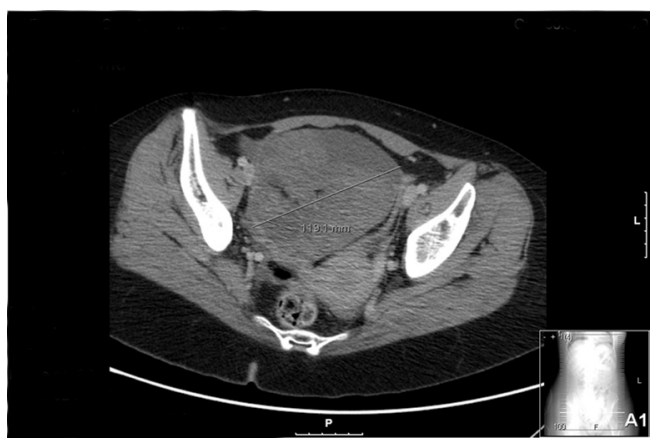

Figure 1. CT scan of the patient revealed a pelvic mass located to the right ovary with $119 \times 132 \mathrm{~mm}$ in dimensions; calcification and fat foci inside suggestive of malignancy namely teratoma. 


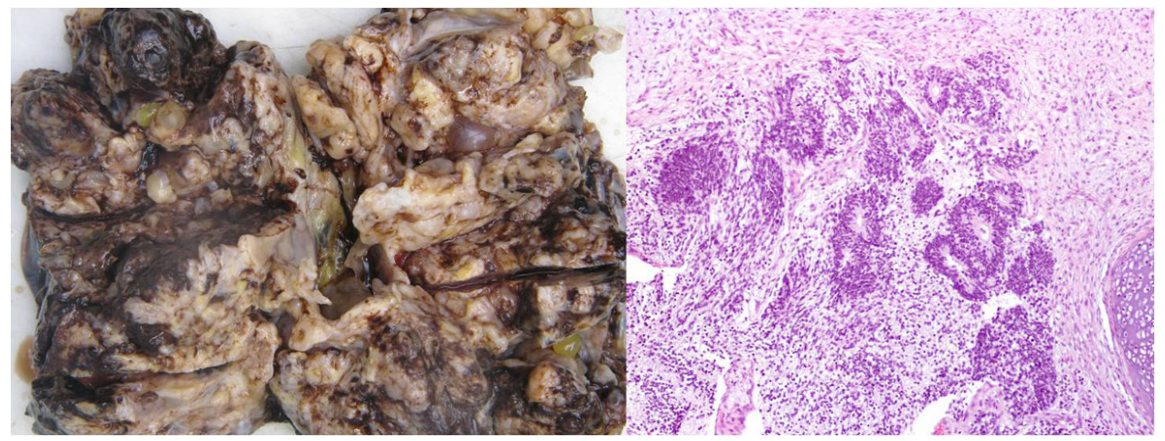

Figure 2. Macrocopic and microscopic analysis of the ovarian tissue revealed the presence of immature mesenchymal elements such as cartilage and adipose tissue. Mature elements such as bone, smooth muscle, keratined epidermis, sweat and sebaceous glands were also seen, as well as respiratory and gastrointestinal type epithelial cells. Findings confirmed the presence of immature, grade 2, ovarian teratoma.

tory hallucination which were treated at home with anti-psychotic drugs but remain unchanged. She was admitted to the psychiatric ward with the diagnosis of inaugural psychosis. On Day 1 her level of consciousness deteriorated followed by a tonic-clonic seizure and was transferred to an intermediate care unit. Complete blood work, with thyroid hormone level determination was unremarkable as was the initial cranial CT scan. A lumbar puncture (LP) was performed and revealed moderate mononuclear pleocytosis (17 cells) with normal protein and glucose levels. Empiric treatment for Herpes simplex virus infection with IV acyclovir was initiated as was phenytoin. During the following 2 days the patient's status fluctuated, with her behaviour changing rapidly from profound catatonia to periods of agitation accompanied by abnormal movements such as leg jerks and oro-lingualfacial dyskinesias. Autonomic instability soon followed with periods of tachycardia and hypertension giving in to periods of bradycardia and hypotension. Hypoventilation ensued. She remained afebrile during this period. On Day 3 acyclovir was stopped after the microbiology of the cerebral spinal fluid was negative for HSV infection. A brain MRI was performed and was also unremarkable. The electroencephalogram (EEG) showed non-specific, slow and disorganised activity, located bilaterally in the fronto-temporal lobes raising the hypothesis among others of limbic encephalitis. Abdominal-pelvic ultrasound followed by CT scan showed a mass in the right ovary with $119 \times 132 \mathrm{~mm}$ in size, with calcification and fat foci inside suggesting the presence of a teratoma (Fig. 1).

A second LP was performed and sampled for the presence among others of antineuronal antibodies. She started immunotherapy with corticosteroids and intravenous immunoglobulin and a complete oophorectomy was performed on day 4. Histopathological analysis confirmed the presence of an immature, grade 2, ovarian teratoma (Fig. 2). After surgery she was transferred to the intensive care unit (ICU) where she remained for the following 30 days of hospital stay.
During her stay in the ICU she received another cycle of corticosteroids and IvIg. Chemotherapy was also initiated with the administration of bleomycin, etoposide and cisplatin. The samples of cerebral spinal fluid and serum that were sent for analysis came out positive for the presence of NMDA receptor antibodies (NR1/NR2 heteromeres).

The patient responded well to the second cycle of immunotherapy and began to recover in the reverse order of symptom development. She was discharged from hospital on day 45 of hospital stay, with no neurological/psychiatric symptoms, a normal neurological examination and with anterograde amnesia starting 10 days before hospital admission up to awaking in the ICU.

\section{Discussion}

Limbic encephalitis (LE) is an inflammatory process involving the hippocampus, amygdalae and orbitofrontal cortex [7]. Patients with these disorders usually present with a constellation of symptoms that manifest in a time-dependent manner: psychiatric disturbances, memory deficits, seizures followed by autonomic instability leading eventually to life support in an intensive care unit or death. After excluding central nervous system infection by neurotropic viruses, as well as use of psychotropic drugs and other metabolic/toxic derangements, a presumptive diagnosis of autoimmune encephalitis (paraneoplastic or non-paraneoplastic in nature) may be assumed $[7,8]$.

In autoimmune mediated LE, MRI maybe unremarkable in more than $50 \%$ of patients, but EEG and CSF show characteristic abnormalities, with the hallmark being the presence of circulating antineuronal antibodies in serum and CSF [6]. In paraneoplastic autoimmune LE, the presence of antibodies directed against tumour tissue cross-react with epitopes of the nervous system, with the clinical outcome being closely related to the type of auto-antibody produced and tumour involved [9]. 
NMDA receptors are located in post-synaptic membranes, and serve as ligand-gated cation channels with major significance for synaptic transmission and plasticity [10]. In anti-NMDAR encephalitis, a rapid, selective and reversible loss of surface NMDAR occurs, with antibody-mediated capping and internalisation, resulting in abrogation in NMDAR-mediated synaptic function $[6,8,9]$. As a result, antibody-mediated decrease in NMDAR predominantly inactivates $\gamma$-amino-butyric acid (GABA)neurons, which express high concentrations of this receptor, leading to disinhibition of excitatory pathways and increase of extracellular glutamate, ensuing in the development of a frontostriatal syndrome characteristic of this encephalitis $[6,10]$. GABA neurons located in brainstem and pons-medullary network are also affected, compromising autonomic stability $[6,10]$.

About $80 \%$ of patients with anti-NMDAR encephalitis are women, predominantly children and young adults [6]. The most frequent underlying tumour is the ovarian teratoma, with age, sex and ethnicity playing an important role in its epidemiology [6]. The presence of anti-NMDAR antibodies in serum and especially in CSF is critical to the diagnosis.

The treatment of these disorders resides in timely initiation of immunotherapy, namely corticosteroids, intravenous immunoglobulin and/or plasma exchange as well as tumour resection and adequate chemotherapy [11]. These patients respond faster to treatment and less frequently need second line immunotherapy namely cyclophosphamide or rituximab than do patients whose diagnosis and subsequent treatment is protracted or who do not have a tumour associated encephalitis [5]. The probability of responding to treatment is also higher in anti-NMDAR encephalitis in comparison with other paraneoplastic encephalitis related with intracellular antigens (anti-Hu for example) [8].

More than three quarters of patients have substantial recovery, with symptoms characteristically regressing in inverse order of development, associated with the decline of antibody CSF titres [12]. Amnesia for the acute phase of the disease is common.

Of increasing importance, as can be seen by the number of recent publications addressing this syndrome [13], antiNMDAR encephalitis should be considered when addressing encephalitis of unknown aetiology, after excluding infectious and metabolic/toxic causes. Its distinguishing clinical picture, accompanied by a normal brain MRI, and the presence of mononuclear pleocytosis in the CSF should raise the suspicion for its diagnosis, and prompt for the search of an occult tumour (usually an ovarian teratoma). Timely initiation of therapy is paramount in the favourable outcome.

\section{Acknowledgement}

The authors would like to thank Professor Josep Dalmau at the Neurology Department of the University of Pennsylvania.

\section{References}

1. Vitaliani R, Mason W, Ances B, Zwerdling T, Jiang Z, Dalmau J. Paraneoplastic encephalitis, psychiatric symptoms, and hypoventilation in ovarian teratoma. Ann Neurol. 2005;58(4):594-604.

2. Dalmau J, Tuzun E, Wu HY, Masjuan J, Rossi JE, Voloschin A, Baehring JM, et al. Paraneoplastic anti-Nmethyl-D-aspartate receptor encephalitis associated with ovarian teratoma. Ann Neurol. 2007;61(1):25-36.

3. Modvig S, Hoi-Hansen CE, Uldall P, Somnier F. [Autoimmune synaptic encephalitis is a disease entity on the rise]. Ugeskr Laeger. 2012;174(21):1452-1456.

4. Pinho J, Rocha J, Rodrigues M, Pereira J, Mare R, Ferreira C, Lourenco E, et al. Diversity in anti-N-methylD-aspartate receptor encephalitis: case-based evidence. Psychiatry Clin Neurosci. 2012;66(2):153-156.

5. Dalmau J, Gleichman AJ, Hughes EG, Rossi JE, Peng X, Lai M, Dessain SK, et al. Anti-NMDA-receptor encephalitis: case series and analysis of the effects of antibodies. Lancet Neurol. 2008;7(12):1091-1098.

6. Dalmau J, Lancaster E, Martinez-Hernandez E, Rosenfeld MR, Balice-Gordon R. Clinical experience and laboratory investigations in patients with anti-NMDAR encephalitis. Lancet Neurol. 2011;10(1):63-74.

7. Lawn ND, Westmoreland BF, Kiely MJ, Lennon VA, Vernino S. Clinical, magnetic resonance imaging, and electroencephalographic findings in paraneoplastic limbic encephalitis. Mayo Clin Proc. 2003;78(11):13631368.

8. Wandinger KP, Saschenbrecker S, Stoecker W, Dalmau J. Anti-NMDA-receptor encephalitis: a severe, multistage, treatable disorder presenting with psychosis. J Neuroimmunol. 2011;231(1-2):86-91.

9. Takahashi Y. Epitope of autoantibodies to N-methyl-Daspartate receptor heteromers in paraneoplastic limbic encephalitis. Ann Neurol. 2008;64(1):110-111; author reply 111-112.

10. Graus F, Saiz A, Dalmau J. Antibodies and neuronal autoimmune disorders of the CNS. J Neurol. 2010;257(4):509-517.

11. Nunez-Enamorado N, Camacho-Salas A, Belda-Hofheinz S, Cordero-Castro C, Simon-De Las Heras R, Saiz-Diaz R, Martinez-Sarries FJ, et al. [Fast and spectacular clinical response to plasmapheresis in a paediatric case of anti-NMDA encephalitis]. Rev Neurol. 2012;54(7):420-424.

12. Irani SR, Bera K, Waters P, Zuliani L, Maxwell S, Zandi MS, Friese MA, et al. N-methyl-D-aspartate antibody encephalitis: temporal progression of clinical and para- 
clinical observations in a predominantly non-paraneoplastic disorder of both sexes. Brain. 2010;133(Pt 6):1655-1667.

13. Mann A, Machado NM, Liu N, Mazin AH, Silver K,
Afzal KI. A multidisciplinary approach to the treatment of anti-NMDA-receptor antibody encephalitis: a case and review of the literature. J Neuropsychiatry Clin Neurosci. 2012;24(2):247-254. 